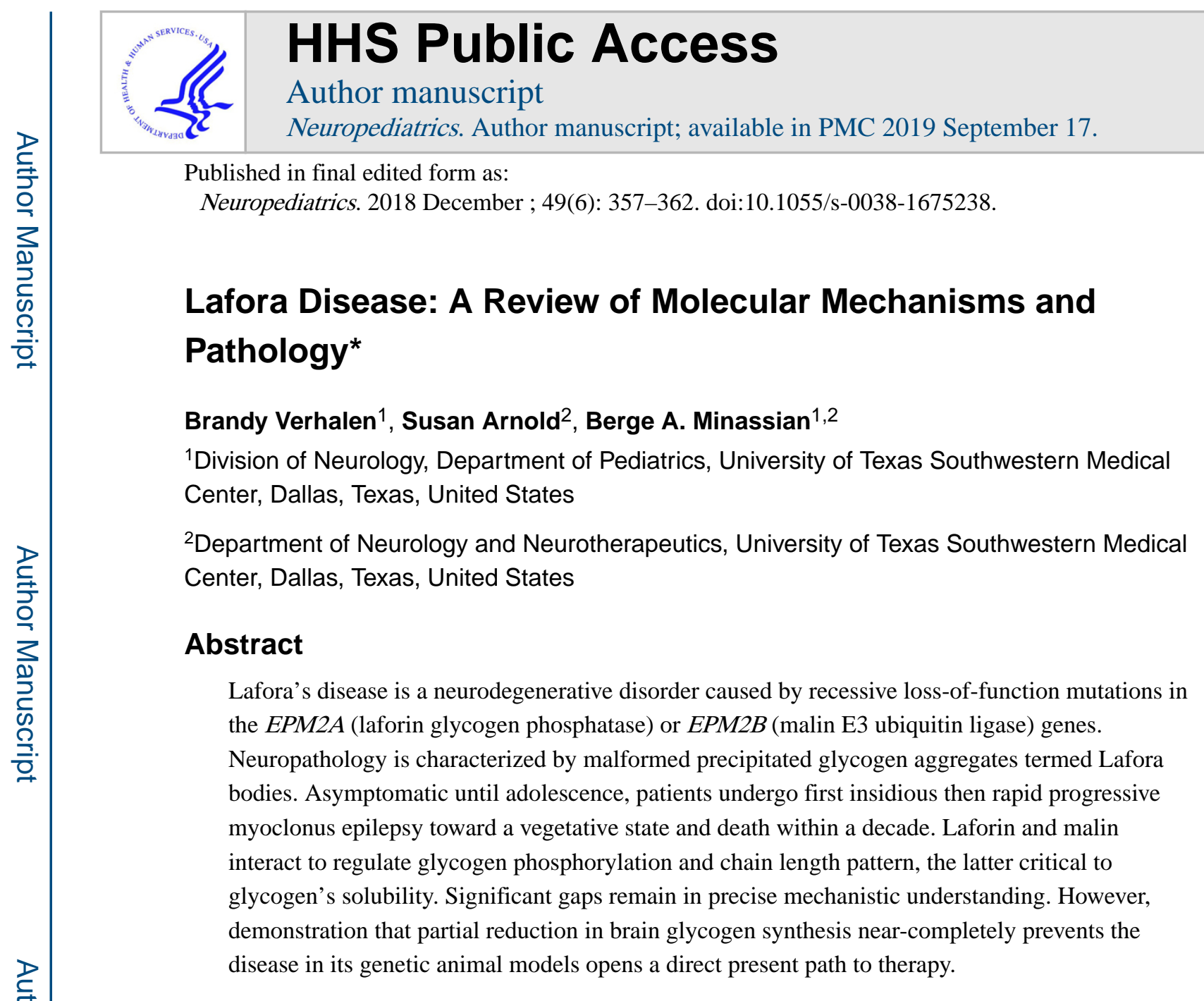

Keywords

Lafora Disease; progressive myoclonus epilepsy; polyglucosan body

\title{
Brief History
}

Lafora disease (LD) is the second identified of the dozen or so progressive myoclonus epilepsies. In the latter part of the first decade of the $20^{\text {th }}$ century, the Spanish neuropathologist Gonzalo Lafora, a student of Cajal, Alzheimer, and Kraeplin, was working near Washington DC in the then Government Hospital for the Insane where he saw the first patient. His descriptions of the neurological features, the recessive inheritance, and the disease course were so complete that they were never really significantly complemented since. Lafora had access to a microscope but not a camera. He studied several autopsy brains

\footnotetext{
* This work has been presented during the 46th SENP (Société Européenne de Neurologie Pédiatrique) meeting, in Barcelona (April $\left.27^{\text {th }}-28^{\text {th }}, 2018\right)$.

Address for correspondence Berge A. Minassian, MD, Division of Neurology, Department of Pediatrics, University of Texas Southwestern Medical Center, 5323 Harry Hines Belvedere, MC9063, Dallas, TX 75390, United States,

(berge.minassian@utsouthwestern.edu).

Conflict of Interest

Dr. Minassian reports grants from National Institutes of Health (NIH) during the conduct of the study; In addition, Dr. Minassian has a licensed Lafora's disease genes patent.
} 
of the patients and was the first to be struck by the presence of profuse massive two-layered spherical structures often occupying the entirety of neuronal cell bodies ( Fig. 1). ${ }^{1}$ These brains were clearly different from those of the patients described in the waning years of the preceding century by Unverricht whose progressive myoclonus epilepsy lacked any obvious neuropathological correlate. ${ }^{2}$ It was over half a century later, while Lafora was still alive and working, that the community of pathologists started calling the disease by his name and the inclusions Lafora's bodies (LB).

\section{Clinical Features}

The typical LD child presents in the approximate middle-third of the second decade of life with vague symptoms of headaches and school difficulties. Soon thereafter myoclonus appears and a generalized tonic-clonic seizure. Given the high frequency of the benign Juvenile Myoclonic Epilepsy (JME), a clinical diagnosis of this epilepsy is invariably first made until successive electroencephalographs (EEG's) show, unlike in JME, a slowing background and spike-wave discharges ( Fig. 2) that do not have the regularity of the latter's spike and polyspike-waves. The patient commonly develops visual hallucinations, usually frightening which have been shown to be both epileptic and psychotic. ${ }^{3}$ After responding to medications (valproic acid [mainstay], perampanel, levetiracetam, zonisamide) initially, seizures and especially the myoclonus become intractable. Atypical and myoclonic absences set in and then become so constant that the young patient's every thought and sentence are constantly interrupted and incomplete. These attacks are both photosensitive and highly affected by emotion. Typically, myoclonus gradually escalates for several weeks until a generalized convulsion occurs (in females usually at menses) following which the myoclonus is greatly improved until the cycle resumes. Within a few years the patient is out of school and is unable to walk mainly because of frequent myoclonic and atonic attacks. Behavioral abnormalities emerge, commonly in the realm of a disinhibited dementia. Awareness is maintained for up to a decade but is then lost often dramatically following an attack of status epilepticus. Sodium channel blocking antiseizure medications, such as phenytoin worsen myoclonus and probably seizures but can be very beneficial in stopping status epilepticus. Most patients pass away in status epilepticus or from aspiration pneumonia secondary to neurological loss of the ability to control secretions. If airways are maintained clear through gastric feeding and attentive suctioning, these patients can survive with little to no responsiveness into the early forties despite the presence of large amounts of LB in skeletal muscle, liver, heart, and other organs. ${ }^{4}$

\section{Pathology}

For many years the abnormal brain deposits defining Alzheimer's disease were thought to be of carbohydrate origin and are called, even today, amyloid (from amylose: starch). They have long since proven to be not of carbohydrate but of protein origin. LB are the true carbohydrate accumulations in neuropathology.

LB in LD are found in all organs that form glycogen. They are insoluble deposits that constantly grow in size and amass at sides of cells without interfering with cell function in hepato-cytes, myocytes, cardiomyocytes, and other cells but not in the brain. Lafora himself, 
and all studies subsequent showed that the larger LB are in neuronal cell bodies usually juxtanuclear. He and others also reported them in shafts of dendrites. In addition to the large somatic LB, there are countless small LB in numerous cell processes. ${ }^{5}$ These were long reported to be neuronal processes and a paradox long existed as to how come neurons which have almost no glycogen and make LB in LD, while astrocytes which are rich in glycogen do not. This mysterious segregation in certain cell types was present in other organs as well, in the liver, LB are in periportal but not perivenous cells. In skeletal muscle they've been reportedtobe in fast-twitch type II but not slow-twitch type I fibers. In eccrine sweat glands, they are in the gland duct cells but not in the secretory cells of the gland. ${ }^{4}$ These cell type specificities suggest particular metabolic properties that might afford important insights into why and how LB form. However, this whole aspect of the disease very recently has entered a state of confusion. It was reported contrary to established dogma that in the LD genetic mouse models the profuse small LB are not in neuronal but rather in astrocytic processes. ${ }^{6}$ However, a simultaneous study of the genetic canine model of LD reported the small LB again in neuronal processes. ${ }^{7}$ This canine paper also reanalyzed the location of LB in skeletal muscle, this time with results contrary to what is reported in the mouse models, ${ }^{8}$ namely that the LB are in slow-twitch type I fibers. ${ }^{7}$ The current confusion obviously requires a careful systematic crass-species reevaluation.

But why is the brain essentially the only affected organ and why with such a severe epilepsy unlike adult-onset neurodegenerative diseases such as Alzheimer's and even most childhood onset neuro degenerative diseases? It has been speculated that the gradual overtaking of synaptic cell processes by LB may underlie the epileptogenesis by escalating disturbance of synaptic function. But is it instead the astrocytic disturbance at synapses as the recent murine studies suggest? It has also been suggested that there is preferential degeneration of inhibitory interneurons in $\mathrm{LD}^{9}$ but this needs further substantiation. It must be noted that whatever the rale of LB in the epilepsy of LD, it is played out in the cortex, unsurprisingly. LB are not found in the long axons of subcortical white matter tracts in contradistinction with another disease exhibiting massive brain carbohydrate accumulations, the clinically amyotrophic lateral sclerosis-like condition known as Adult Polyglucosan Body Disease (APBD).

\section{Glycogen, Polyglucosans, and Lafora's Bodies}

One of the principle ways in which animals store energy is in chains of al-4 linked glucose molecules. The linkage is mediated by the enzyme glycogen synthase (GS). However, polyglucan chains of a dozen glucoses long or longer form double helices, extrude water, and precipitate. So then how does glycogen with up to 55,000 glucose units remain eminently soluble? This feat of evolution is achieved through regular branching. Every chain dozen or so glucose units in length is branched by the enzyme glycogen branching enzyme (GBE) through formation of al-6 interglucosidic bonds. As such, the polyglucan chains are directed away from each other preventing them from wrapping around each other. The countless branches of the massive molecule are interspersed with water, and glycogen is thus soluble and available for rapid digestion when energy is needed. ${ }^{10}$ Complete GBE deficiency is not compatible with life but partial GBE deficiency results in poorly branched glycogen called polyglucosan which precipitates and accumulates in various organs. If the 
GBE deficiency is severe, the accumulations are massive and affected patients die of liver failure (Andersen's disease). If the deficiency is mild, the amounts of polyglucosans formed and accumulating are relatively small and do not harm heart or liver. These patients live into their 50s in good health but then develop the neurological disease APBD due to accumulation of polyglucosans in subcortical neuronal long axons. As mentioned, APBD patients have upper and lower motor neuron disease likely due to the progressive axonopathy resulting from the clogging of axons by the ever-growing polyglucosan bodies. They do not suffer epilepsy. ${ }^{11,12}$

The LB in LD are similar to the polyglucosan bodies of APBD. They are similarly composed of poorly branched and therefore insoluble glycogen (polyglucosans) which accumulate over time. LD polyglucosans differ in one important way from GBE polyglucosans, namely, in that they possess a large amount of covalently bound phosphate. Why LB are not in long axons but instead in neuronal cell bodies and in neuronal (or astrocytic) short processes are unknown. LD and the polyglucosans that make up LB are not due to GBE deficiency nor are they due to GS overactivity. ${ }^{13-16}$

\section{Genetic Causes and Basic Mechanisms}

Until the LD genes were discovered in the latter years of the 20th century, the diagnosis of LD relied on biopsies, initially of the brain, and later liver, skeletal muscle, and eventually skin. As mentioned, LB are found in skin eccrine sweat gland ducts. They are also found in the myoepithelia of apocrine glands ( Fig. 3). Today, the diagnosis of LD is based on biallelic mutation identification in either the EPM2A or $E P M 2 B$ gene. The vast majority of mutations are loss-of-function and as such the disease is rather uniform across the two genes and most mutations. Few rare mutations result in a milder, more slowly progressive phenotype. ${ }^{17,18}$

$E P M 2 A$ encodes the glycogen phosphatase laforin which dephosphorylates glycogen. $E P M 2 B$ encodes the ubiquitin E3 ligase malin whose function remains mysterious, except for the fact that it is a strong interacting partner of Iaforin. The LD glycogen hyperphosphorylation mentioned above applies equally to Iaforin or malin deficient LD. 13,16,19,20 There are presently two divergent hypotheses of polyglucosan formation in LD based on yet unreconciled conflicting data. One hypothesis posits that phosphates are added to glycogen as an undesirable side reaction of the GS enzyme and that these phosphates lead to glycogen precipitation and malstructuring through yet to be clarified mechanisms. This hypothesis is mainly supported by laforin's unequivocal role as a glycogen phosphatase and by evidence that such a GS phosphate-adding side reaction does occur. ${ }^{21,22}$ The other hypothesis considers glycogen phosphorylation as a desirable event through a yet unidentified kinase similar to what occurs in plant starches. In plants, the insoluble starch is made soluble at the surface through phosphorylation with the added phosphates through their high charges separating glucan chains. In this hypothesis, every once in a while during glycogen synthesis a glycogen chain is overextended by GS. Such a chain would be phosphorylated to prevent it from wrapping around a neighbor chain. This would attract laforin which would not only bind the phosphoglucan but via malin would ubiquitinate and inhibit GS. Laforin would then remove the phosphate which in plants is known to be 
required for chain digestion. One important support for this hypothesis is that glycogen in laforin-deficient mice overexpressing phosphatase-inactivated laforin is hyperphosphorylated but is neither long-chained nor insoluble and these mice no longer form LB. On the other hand, glycogen in malin-deficient mice overexpressing phospha-taseinactivated laforin is hyperphosphorylated and long-chained and these mice continue to form LB. ${ }^{14,15,23,24}$

\section{Treatment}

First and foremost LD is a personal and family catastrophe in those affected. The aggressive yet protracted neurodegeneration, with all its physical and psychological trauma including the fear of younger children being at risk, comprises a nearly unbearable toll. As such the overall family unit requires all the support that can possibly be extended.

For the epilepsy, antiseizure medications are helpful in the early phases of the disease and probably life-sustaining and extending in later phases but certainly far from adequate. The known genetic causation and the progress in gene therapy methods in recent years may make gene replacement a strong option in this and in all single gene defect neurodegenerative diseases. The ketogenic diet has been tried but without success, albeit in relatively advanced cases. ${ }^{25}$ The question remains whether this approach would slow or delay disease progression in newly diagnosed patients not only through a general antiepileptic impact but also possibly by reducing the substrate of glycogen (and polyglucosans) in the brain.

The most exciting approach to therapy at the moment comes from results in the LD mouse models. It has been shown that a mere genetic 50\% reduction in GS activity in the brain dramatically reduces to near completely eliminating LB. This is accompanied with absence of the neurological abnormalities and neurodegeneration that are otherwise present in these mice. ${ }^{26-29}$ Work is actively underway in multiple laboratories to identify inhibitors of GS at both the mRNA (messenger ribonucleic acid) and protein levels.

It is this rare disease that taught us that there is more to glycogen structure than a balance of activities between GS and GBE. Precisely what the role of the laforin-malin system is in regulating the solubility of glycogen remains tantalizing. This understanding will not only enhance general understanding of metabolism but also specifically as it relates to brain function and epilepsy. It will also allow insights that will help devise treatments for LD. Meanwhile, the progress to date allows a highly promising path forward, even now, through partial brain GS downregulation to help treat this catastrophic epilepsy.

\section{Acknowledgments}

This work was supported by the National Institute of Neurological Disorders and Stroke of the National Institutes of Health (NIH) under award number P01 NS097197. Berge A Minassian holds the University of Texas Southwestern Jimmy Elizabeth Westcott Chair in Pediatric Neurology.

\section{References}

1. Lafora GR, Glueck B. Beitrag zur Histopathologie der myoklo-nischen Epilepsie. Zeitschrift für die gesamte Neurologie und Psychiatrie 1911;6(Suppl 1): 1-14 
2. Unverricht H Die Myoclonie. Leipzig, Vienna: Franz Deuticke; 1891

3. Andrade DM, del Campo JM, Moro E, Minassian BA, Wennberg RA. Nonepilepticvisual hallucinations in Lafora disease. Neurology 2005;64(07):1311-1312 [PubMed: 15824378]

4. Turnbull J, Tiberia E, Striano P, et al. Lafora disease. Epileptic Disord 2016;18(S2):38-62 [PubMed: 27702709]

5. Van Heycop Ten Ham MW. Lafora disease, a form of progressive myoclonus epilepsy. Handb Clin Neurol 1975;15:382-422

6. Rubio-Villena C, Viana R, Bonet J, et al. Astrocytes: new players in progressive myoclonus epilepsy of Lafora type. Hum Mol Genet 2018;27(07):1290-1300 [PubMed: 29408991]

7. Chambers JK, Thongtharb A, Shiga T, et al. Accumulation of Laforin and other related proteins in canine Lafora disease with EPM2B repeat expansion. Vet Pathol 2018;55(04):543-551 [PubMed: 29444631]

8. Turnbull J, Girard JM, Pencea N, et al. Lafora bodies in skeletal muscle are fiber type specific. Neurology 2011;76(19):1674-1676 [PubMed: 21451149]

9. Ortolano S, Vieitez I, Agis-Balboa RC, Spuch C. Loss of GABAergic cortical neurons underlies the neuropathology of Lafora disease. Mol Brain 2014;7:7 [PubMed: 24472629]

10. Roach PJ. Glycogen and its metabolism. CurrMol Med 2002;2(02): 101-120

11. Lossos A, Meiner Z, Barash V, et al. Adult polyglucosan body disease in Ashkenazi Jewish patients carrying the Tyr329Ser mutation in the glycogen-branching enzyme gene. Ann Neurol 1998;44(06):867-872 [PubMed: 9851430]

12. Robitaille Y, Carpenter S, Karpati G, DiMauro SD. A distinct form of adult polyglucosan body disease with massive involvement of central and peripheral neuronal processes and astrocytes: a report of four cases and a review of the occurrence of polyglucosan bodies in other conditions such as Lafora's disease and normal ageing. Brain 1980;103(02):315-336 [PubMed: 6249438]

13. DePaoli-Roach AA, Tagliabracci VS, Segvich DM, Meyer CM, Irimia JM, Roach PJ. Genetic depletion of the malin E3 ubiquitin ligase in mice leads to lafora bodies and the accumulation of insoluble laforin. J BiolChem 2010;285(33):25372-25381

14. Nitschke F, Sullivan MA, Wang P, et al. Abnormal glycogen chain length pattern, not hyperphosphorylation, is critical in Lafora disease. EMBO Mol Med 2017;9(07):906-917 [PubMed: 28536304]

15. Sullivan MA, Nitschke S, Steup M, Minassian BA, Nitschke F. Pathogenesis of Lafora disease: transition of soluble glycogen to insoluble polyglucosan. Int J MolSci 2017;18(08):E1743

16. Tagliabracci VS, Girard JM, Segvich D, et al. Abnormal metabolism of glycogen phosphate as a cause for Lafora disease. J BiolChem 2008;283(49):33816-33825

17. Chan EM, Young EJ, Ianzano L, et al. Mutations in NHLRC1 cause progressive myoclonus epilepsy. Nat Genet 2003;35(02):125-127 [PubMed: 12958597]

18. Minassian BA, Lee JR, Herbrick JA, et al. Mutations in a gene encoding a novel protein tyrosine phosphatase cause progressive myoclonus epilepsy. Nat Genet 1998;20(02):171-174 [PubMed: 9771710]

19. Turnbull J, Wang P, Girard JM,et al. Glycogen hyperphosphorylation underlies lafora body formation. Ann Neurol 2010;68(06):925-933 [PubMed: 21077101]

20. Worby CA, Gentry MS, Dixon JE. Laforin, a dual specificity phosphatase that dephosphorylates complex carbohydrates. J BiolChem 2006;281(41):30412-30418

21. Contreras CJ, Segvich DM, Mahalingan K, et al. Incorporation of phosphate into glycogen by glycogen synthase. Arch Biochem-Biophys 2016;597:21-29 [PubMed: 27036853]

22. Tagliabracci VS, Heiss C, Karthik C, et al. Phosphate incorporation during glycogen synthesis and Lafora disease. Cell Metab 2011; 13 (03):274-282 [PubMed: 21356517]

23. Gayarre J, Duran-Trio L, Criado Garcia O, et al. The phosphatase activity of laforin is dispensable to rescue Epm2a-/- mice from Lafora disease. Brain 2014;137(Pt 3):806-818 [PubMed: 24430976]

24. Nitschke F, Wang P, Schmieder P, et al. Hyperphosphorylation of glucosyl C6 carbons and altered structure of glycogen in the neurodegenerative epilepsy Lafora disease. Cell Metab 2013;17 (05): 756-767 [PubMed: 23663739] 
25. Cardinali S, Canafoglia L, Bertoli S, et al. A pilot study ofa ketogenic diet in patients with Lafora body disease. Epilepsy Res 2006;69 (02):129-134 [PubMed: 16504479]

26. Duran J, Gruart A, García-Rocha M, Delgado-García JM, Guinovart JJ. Glycogen accumulation underlies neurodegeneration and autophagy impairment in Lafora disease. Hum Mol Genet 2014; 23(12):3147-3156 [PubMed: 24452334]

27. Pederson BA,Turnbull J, Epp JR, et al. Inhibiting glycogen synthesis prevents Lafora disease in a mouse model. Ann Neurol 2013;74 (02):297-300 [PubMed: 23913475]

28. Turnbull J, DePaoli-Roach AA, Zhao X, et al. PTG depletion removes Lafora bodies and rescues the fatal epilepsy of Lafora disease. PLoS Genet 2011;7(04):e1002037 [PubMed: 21552327]

29. Turnbull J, EppJR Goldsmith D, et al. PTG protein depletion rescues malin-deficient Lafora disease in mouse. Ann Neurol 2014;75(03): 442-446 [PubMed: 24419970] 


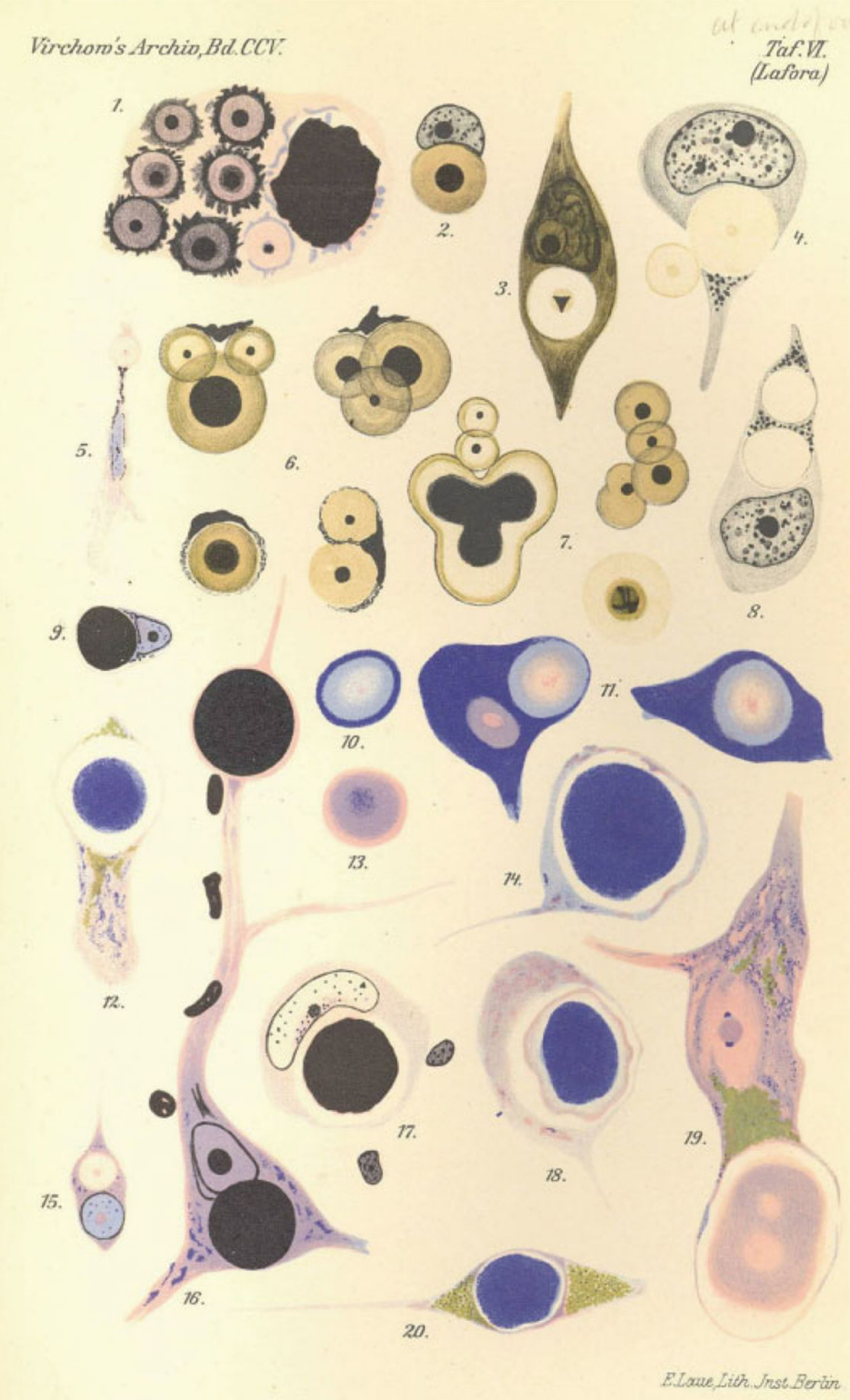

Fig. 1.

Lafora's original drawings of Lafora bodies. 


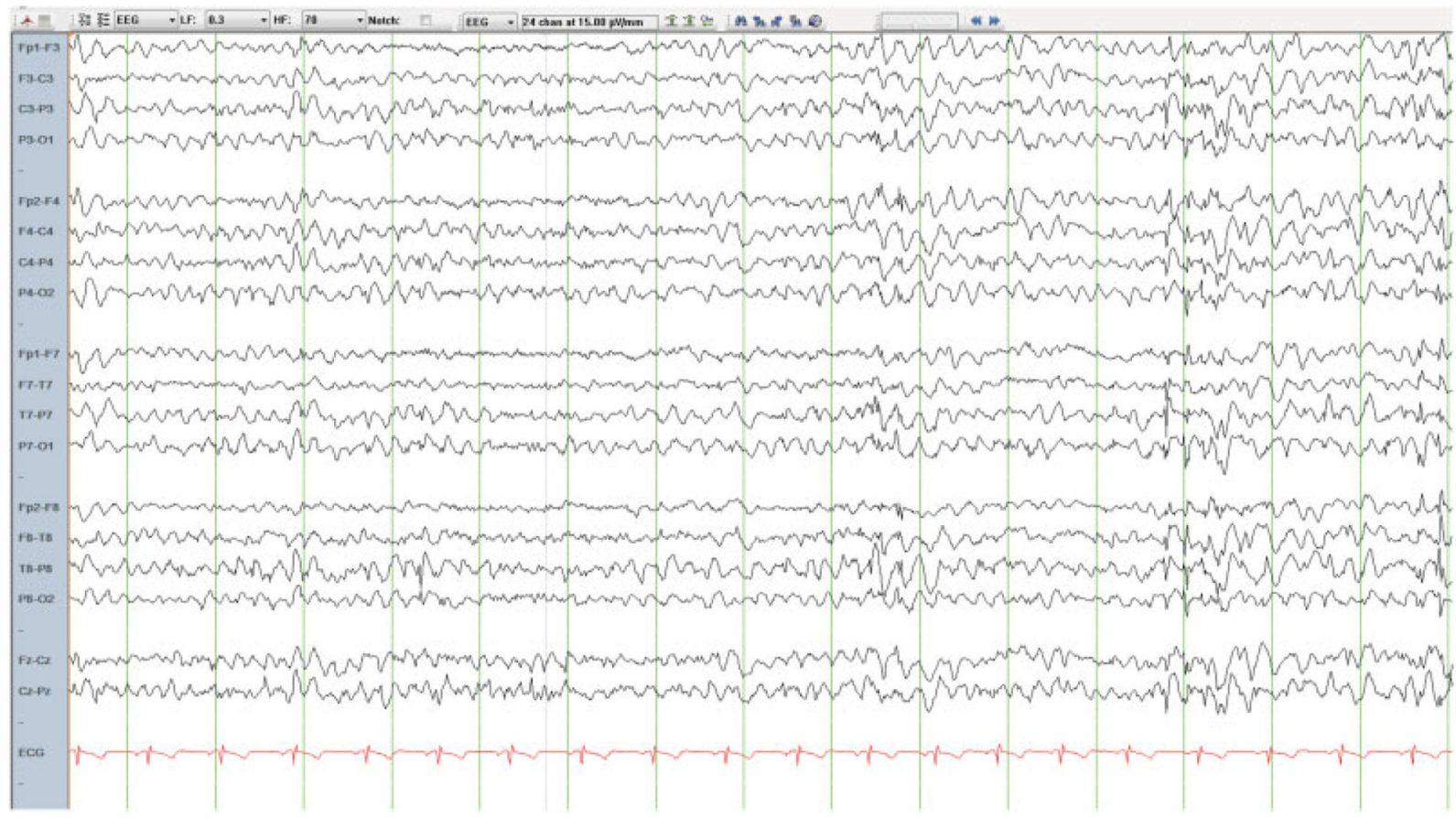

Fig. 2.

Wakeful EEC in a 17-year-old patient with LD and mutations in the EPM2A gene. Note the slow background and irregular generalized spike-wave discharges, e.g., in the last 4 seconds of the EEC page. 


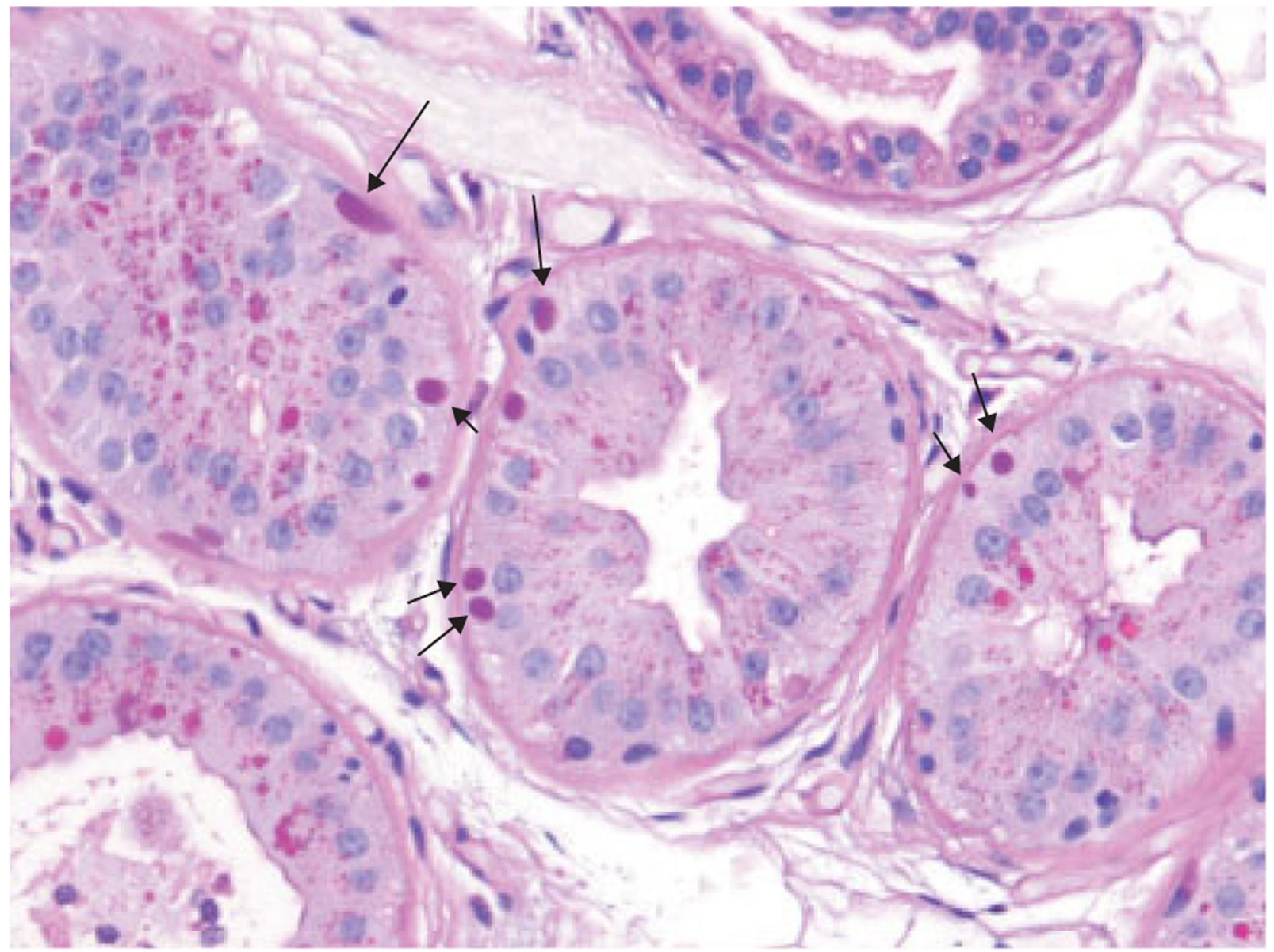

Fig. 3.

Skin biopsy from the same patient as above with periodic acid-Schiff staining. Arrows indicate pathognomonic Lafora bodies (LB) in the myoepithelia (at the bases) of apocrine sweat glands. Note, similarly stained structures near gland lumens(e.g., in the gland at the bottom left corner of the image). Those are the normal secretory products of these glands and are not LB. They are a common source of obviously highly undesired false-positive diagnosis in patients undergoing skin biopsy as part of a workup for progressive myoclonus epilepsy. 